This is an Accepted Manuscript of an article published by Taylor \& Francis in Journal of Interprofessional Care on 20/12/16, available online:

http://www.tandfonline.com/10.1080/13561820.2016.1249281. 


\title{
Expanding Pharmacy Roles and the Interprofessional Experience in Primary Health Care: \\ A Qualitative Study
}

\begin{abstract}
The pharmacist role is undergoing significant changes which are reshaping the way primary health care is delivered throughout England. Due to increased physician workload and focus on primary health care, the pharmacist role has expanded to provide enhanced patient services, integrating into general practice (GP) settings and working more closely as a member of the healthcare team. However, the experiences of pharmacists and team members are yet to be explored. The proposed study aims to explore the experiences, thoughts, and perceptions of a purposive sample of pharmacists, physicians, and nurses working in 10 GP clinics throughout the southeast of England. Interprofessional relationships, power dynamics, changing professional roles, and barriers and facilitators to the integration of the pharmacist role will be explored. An exploratory multiple case study design will be used to investigate interprofessional experiences within and between clinics. In depth interviews will be completed with each participant. A thematic analysis will identify themes and patterns from the interview data. Results are expected to produce recommendations to help facilitate the integration of pharmacists in their new role and will have implications for interprofessional collaboration and interprofessional education which are important for delivering safe and effective care.
\end{abstract}

Keywords: Pharmacist, Expanded Roles, Interprofessional Collaboration, Teamwork, Qualitative Method 


\section{Introduction}

The pharmacist role is undergoing significant changes which are reshaping the way primary health care is delivered throughout England. In response to an aging population, increased physician workload and a greater focus on primary health care, the pharmacist role has expanded to provide enhanced patient services (Mossialos et al., 2015; NHS England, 2015). Enhanced services provided by qualified pharmacists include screening for chronic disease, providing smoking cessation, treating minor ailments, and prescribing certain medications (Mossialos et al., 2015). Recently, pharmacists have begun integrating their expanded roles into general practice (GP) settings, providing more patient care and working closely as a member of the healthcare team. A large pilot study funded by the National Health Service (NHS) is underway to integrate 403 pharmacists into GP settings (NHS England, 2015). However, despite the potential benefits of integrating clinical pharmacists into GP settings, the experiences of the pharmacist and other members of the healthcare team are yet to be explored.

This paper presents details of a proposed study which aims to explore the experiences, thoughts, and perceptions of pharmacists, physicians and nurses working in 10 GP clinics throughout the south east of England. Additionally, interprofessional relationships, power dynamics, changing interprofessional roles, and barriers and facilitators to the integration of the expanded pharmacist role are explored.

\section{Methods}

An exploratory multiple case study design (Yin, 2003) will be used to explore how multiple health care providers experience working within a GP clinic utilizing the expanded pharmacist role. With a multiple case study, several cases are analyzed in order to understand the 
differences and similarities between cases (Yin, 2003). Using this design, interprofessional experiences will be analyzed within and between clinics to explore interprofessional relationships, changing interprofessional roles, and power differentials under different contextual conditions. Furthermore, barriers as well as factors that enhance the utilization of the expanded pharmacist role will be explored.

\section{Data collection}

The proposed study will be conducted within 10 GP clinics throughout the south east of England where pharmacists are integrating into their practice. A purposive snowball sample from a population of 30 clinics will be used to invite participants. Semi-structured, in-depth interviews will be conducted with one nurse, pharmacist, and physician from each clinic, aiming for a total of 30 participants. Use of multiple interviewers engaging with a variety of health care providers will help gain an in-depth understanding of the interprofessional experience from several perspectives.

\section{Data analysis}

A thematic analysis will be conducted to search for themes and patterns. Data collection and analysis will occur simultaneously throughout the research project (Gale et al., 2013; Yin, 2003). Interviews will be audio-taped and transcribed verbatim and line-by-line coding will be conducted to search for themes using both inductive and deductive approaches. While the researchers will be searching for specific themes previously selected from the literature, such as interprofessional relationships, power dynamics, and changing roles, the researchers will also remain open to other themes that may be identified from the interview data. An analytical framework will be developed with the input of all researchers involved, where coded data will be grouped into categories which will be used to compare with subsequent transcripts (Gale et al., 
2013). Data will be analyzed using NVivo qualitative data analysis Software (QSR International Pty Ltd. Version 10, 2014) to help organize the coding, categorizing, and charting of data into an analytic framework. Lastly, characteristics and differences between the data will be identified to explore the relationships of themes related to interprofessional experiences between and within GP clinics.

\section{Ethical considerations}

The research study will commence once it has been approved by the relevant ethical review board. Participation in the study is voluntary and the identity of the participants will be kept private and confidential. All data will be stored in a secure and confidential manner.

\section{Discussion}

Lack of communication and awareness of interprofessional roles has been shown to reinforce power differentials within the interprofessional team, leading to a lack of participation in interprofessional education and decreased interprofessional collaboration (Baker, Egan-Lee, Martimianakis, \& Reeves, 2011). Lack of role awareness, poor communication, and insufficient collaboration have been associated with a decreased acceptance of pharmacy roles within primary health care teams in the United States and Canada (Patterson et al., 2015; Schindel et al., 2016). Schindel et al. (2016) found that collaboration with other health care professionals was essential because the pharmacist role often overlapped with the role of other health care professionals, which, at times, led to conflict between team members. Therefore, there is a need to revisit the pharmacist role as their scope of practice changes (Schnidel et al., 2016). Since the expanded pharmacist role in GP clinics is relatively new in England and around the world, very few studies have been conducted on this topic. 
The proposed study is expected to assist health care educators and professionals to gain a better understanding of the experience of pharmacists working in expanded roles and their impact on the interprofessional team. Barriers and facilitators to the integration of pharmacists into GP will be identified. It is anticipated that the analysis and findings will provide a framework from which to make recommendations for pharmacists, physicians, and nurses working in GP clinics to help facilitate the integration of pharmacists into their practice. Additionally, results will be used to inform pharmacy educators who are designing curriculums for pharmacists so they are better prepared for this role. Lastly, results and recommendations will be made for those incorporating or facilitating interprofessional education into their curriculum or professional practice with consideration of the changing pharmacist role.

The results of the proposed study are expected to generate a series of key implications for interprofessional collaboration and education, which impacts the delivery of safe and effective healthcare. It is important for other healthcare professionals to be aware of the changing roles of pharmacists and other team members as well as how their own role is affected within the interprofessional team and the impact this has on quality of patient care.

\section{Declaration of interest}

The authors report no conflicts of interest. The authors alone are responsible for the content and writing of the article. 


\section{References}

Baker, L., Egan-Lee. E, Martimianakis, M. A., \& Reeves, S. (2011). Relationships of power: Implications for interprofessional education. Journal of Interprofessional Care, 25(2), 98-104. doi: 10.3109/13561820.2010.505350

Gale, N. K., Heath, G., Cameron, E., Rashid, S., \& Redwood, S. (2013). Using the framework method for the analysis of qualitative data in multi-disciplinary health research. $B M C$ Medical Research Methodology, 13(117), 1-8. doi: 0.1186/1471-2288-13-117

Mossialos, E., Courtin, E., Naci, H., Benrimoj, S., Bouvy, M., Farris, K., ... Sketris, I. (2015). From "retailers" to health care providers: Transforming the role of community pharmacists in chronic disease management. Health Policy, 119(5), 628-639. doi: 10.1016/j.healthpol.2015.02. 007

NHS England. (November, 2015). Clinical pharmacists in General Practice pilot: Announcement of successful sites FAQs. Retrieved from https://www.england.nhs.uk/ commissioning/wp-content/uploads/sites/12/2015/11/clinical-pharm-gp-faq-v3.pdf

Patterson, B. J., Solimeo, S. L., Stewart, K. R., Rosenthal, G. E., Kaboli, P. J., \& Lund, B. C. (2015). Perception of pharmacists' integration into patient-centered medical home teams. Research in Social and Administrative Pharmacy, 11(1), 85-95. doi: 10.1016/j.sapharm. 2014.05.005

QSR International Pty Ltd. (2014). NVivo qualitative data analysis software, Version 10

Schindel, T. J., Yuksel, N., Breault, R., Daniels, J., Varnhagen, S., \& Hughes, C. A. (2016). Perceptions of pharmacists' roles in the era of expanding scopes of practice. Research in Social and Administrative Pharmacy, (in press), 1-14. doi: 10.1016/j.sapharm.2016.02. 007 
Yin, R. K. (2003). Case study research: Design and methods (3rd ed.). Thousand Oaks, CA: Sage Publications 